\title{
The Machine Learning journal: 250 issues and counting
}

\author{
Peter A. Flach
}

Published online: 24 September 2010

(C) The Author(s) 2010

This issue of the Machine Learning journal is the 250th published since the start of the journal in March 1986. For the first five years, four issues were published annually; in 1991 this was increased to six issues, and to eight the following year. From 1993 onwards, the Machine Learning journal publishes 12 monthly issues in four volumes annually. As I only started my job as Editor-in-Chief in July this year, I cannot claim credit for this remarkable and sustained output, which is largely due to the hard work of my predecessors: Pat Langley (1986-9), Jaime Carbonell (1989-92), Tom Dietterich (1993-8), Rob Holte (1998-2003) and Foster Provost (2004-10). To mark the 25th anniversary of the journal in Mach 2011, we will hear from some of them in the coming year as they look back on their time at the helm of the journal, commenting on what was happening in the field back then and what lessons we can learn for machine learning today and tomorrow.

I would like to take this opportunity to express special thanks to my immediate predecessor, Foster Provost. Not only has Foster been the longest serving editor of MLj to date (six and a half years), but during this period he has overseen a number of improvements in the MLj publishing model that benefit the entire community. For example, authors now retain copyright to their papers published in MLj. Furthermore, as soon as corrected proofs are received from the authors, papers scheduled to appear in print are available from Springer's $\mathrm{MLj}$ website (Online First). Springer actively promotes MLj and has provided increased opportunities for freely accessing back issues online via numerous promotional activities. It is also worth mentioning that the journal financially sponsors best student-paper awards at some of the major machine learning conferences, including ICML, ECML, NIPS, COLT and ILP.

With the journal being in such good shape, I do not have any major changes in store at the moment. Having said that, I do believe that there are opportunities to further support and streamline the reviewing process. A key issue here is to make sure that papers are being sent out to the right reviewers. At Bristol we have developed a system that

P.A. Flach $(\bowtie)$

Intelligent Systems Laboratory, University of Bristol, Bristol, UK

e-mail: Peter.Flach@bristol.ac.uk 
matches an abstract or full paper to a set of potential reviewers represented by their publications as recorded in the DBLP online bibliography, and once this system is sufficiently refined we will investigate how to incorporate it into the MLj workflow (see demo at http://subsift.ilrt.bris.ac.uk/demo/sift ). I am also working with our Action Editors to obtain a comprehensive list of keywords and subject areas that can further aid this matching process. I would welcome any suggestions from our readership.

In the meantime, I hope you enjoy reading this 250th issue of the Machine Learning journal, and please stay tuned for 25 th anniversary activities.

Editor-in-Chief 